\title{
FACTIBILIDAD TÉCNICA Y FINANCIERA DEUN MODELO DE CREDIT SCORING PARA LAS ENTIDADES \\ DE AHORRO Y CRÉDITO POPULAR
}

José Francisco Martínez Sánchez María Teresa Martínez Palacios*

(Recibido: noviembre 2014/Aceptado: marzo 2015)

\section{Resumen}

El sistema bancario actual no cubre las necesidades de los servicios financieros y en particular las de los créditos a los sectores más desfavorecidos de la sociedad. La presencia bancaria se localiza fundamentalmente en las ciudades y regiones con actividad económica importante. Para atender a estos sectores excluidos se han creado de forma natural, pero sin supervisión ni soporte de la autoridad, entidades financieras como las cajas populares, cooperativas, sociedades financieras populares, entre otras, que en conjunto se denominan entidades de ahorro y crédito popular. Sin embargo, la mayoría de este tipo de instituciones no son reconocidas ni supervisadas por la Comisión Nacional Bancaria y de Valores (CNBV), lo cual se traduce en riesgos tanto para los usuarios de los servicios como para las instituciones financieras, destacando las ineficiencias en sus procesos de otorgamiento de créditos, ya que las decisiones de aceptar o no una solicitud de crédito se sustenta en el conocimiento, experiencia y criterio del analista de crédito. Este trabajo presenta un análisis de factibilidad de un proceso integral para el análisis y evaluación de las solicitudes de los micro créditos, soportada por un modelo de línea de crédito, tanto en términos de costo-eficiencia para las entidades de ahorro y crédito como en términos de costo-beneficio para el proveedor de los servicios de

* Profesor-investigador de la Universidad Autónoma del Estado de Hidalgo. Escuela Superior de Apan, Carretera Apan-Calpulalpan, Km. 8, Chimalpa Tlalayote s/n, colonia Chimalpa, Apan, Hgo., México, CP. 43900. Correo electrónico:<marzan67@hotmail.com>. 
evaluación de solicitudes de crédito. Como se mostrará en el desarrollo del trabajo un modelo de esta naturaleza hace más eficiente el proceso crediticio a un costo razonable y es una inversión rentable para el proveedor de servicios de línea de crédito.

\section{Abstract}

The current banking system does not meet the needs of financial services, particularly credit to the poorest sectors of society, the banking presence are mainly located in cities and regions with important economic activity, to attend to these excluded sectors have created naturally without supervision and support of the authorities, financial institutions such as credit unions, cooperatives, popular financial companies, among others, called savings banks and loan altogether. However, most of these institutions are not recognized or supervised by the $\mathrm{CNBV}$, which translates into risk for users of services to financial institutions, highlighting the inefficiencies in their lending processes, and decisions to accept or reject a credit application is based on knowledge, experience and judgment of the loan officer. This paper presents a feasibility analysis in terms of cost-efficiency for savings and loan institutions and in terms of cost-benefit to the service provider assessment of loan applications. As will be shown in the development of a model work of this nature streamlines the lending process at minimal cost and is a worthwhile investment for the provider of credit scoring.

Keywords: ICT, economic growth, stochastic optimal control. JEL classification: G31, G32, O38.

\section{Introducción}

En el mes de junio de 2001 fue publicada en el Diario Oficial de la Federación la Ley de Ahorro y Crédito Popular alineado a las mejores prácticas en materia de administración de riesgos crediticios bancarios (Pagos, 2004), mediante la cual se creó la figura de las Entidades de Ahorro y Crédito Popular (EACP), y en donde se contemplaron los siguientes objetivos generales:

a) Regular, promover y facilitar la captación de fondos o recursos monetarios y su colocación mediante préstamos o créditos u otras operaciones por parte de las sociedades financieras populares, 
sociedades financieras comunitarias; así como, los organismos de integración financiera rural;

b) Regular, promover y facilitar las actividades y operaciones de las sociedades financieras populares, sociedades financieras comunitarias y los organismos de integración financiera rural, así como su sano y equilibrado desarrollo;

c) Proteger los intereses de sus clientes, y

d) Establecer los términos en los que el Estado ejercerá la rectoría de las referidas sociedades financieras populares en términos de la ley.

La Ley de Ahorro y Crédito Popular, Comisión Nacional Bancaria y de Valores (CNBV) 2001, busca regular las dos figuras más importantes que deberán adoptar las EACP. Estas figuras son las Sociedades Cooperativas de Ahorro y Préstamo (SCAP), y las Sociedades Financieras Populares (SOFIPO). Una SOFIPO es una entidad que forma parte del sistema financiero mexicano, creada para atender el mercado de micro-finanzas, este trabajo se enfoca a las SOFIPO.

Una SOFIPO está facultada para recibir depósitos, préstamos y créditos de bancos, fideicomisos públicos y organismos internacionales, afores, aseguradoras y afianzadoras; puede expedir y operar tarjetas de débito y prepagadas, otorgar préstamos y créditos a sus clientes, ser transmisor de dinero (órdenes de pago y transferencias), recibir pagos por cuenta de terceros, realizar compra venta de divisas en ventanilla por cuenta de terceros, distribuir seguros, fianzas y recursos de programas gubernamentales.

Las SOFIPO están sujetas a la supervisión de la CNBV, la que tiene todas las facultades que en materia de inspección y vigilancia le confiere las leyes aplicables. Estas facultades pueden ser ejercidas directamente por la CNBV y de manera auxiliar por las federaciones, las cuales se constituyen exclusivamente con la agrupación voluntaria de las SOFIPO; y son autorizadas y supervisadas por la CNBV. Las federaciones son instituciones de interés público, con personalidad jurídica y patrimonio propios, sin fines lucrativos.

Adicionalmente, en diciembre de 2006, se expiden las disposiciones de carácter general aplicables a las entidades de ahorro y crédito popular y organismos de integración a que se refiere la Ley de Ahorro y Crédito Popular, y en ellas se establecen los requisitos que deberán reunir las solicitudes o requerimientos de información que formulen las autoridades a que 
se refiere esta Ley, a efecto de que las SOFIPO requeridas estén en aptitud de identificar, localizar y aportar las noticias o información solicitadas.

Bajo este modelo de operación, las instituciones encargadas y autorizadas por la CNBV para fungir como agentes financieros para captar recursos y otorgar este tipo de créditos enfrentan diversos retos para asegurar su viabilidad y existencia. Uno de los retos principales a enfrentar por este sector es el capital, toda vez que este tipo de entidades no crece porque la base del accionariado acota sus aportaciones. Y es que, para apalancar el crecimiento de cualquier intermediario financiero se requiere un mayor nivel de capital para generar un importante volumen de negocio y un reconocimiento como alternativa de ahorro y crédito por parte de la sociedad.

Otro de los retos para el fortalecimiento de las SOFIPO frente al nuevo entorno económico, es contar con un mecanismo de protección, pues es primordial y relevante para que las personas que confían sus recursos en estos vehículos tengan la certeza que hay un esfuerzo institucional de proteger sus ahorros.

Por último, existe el factor de la innovación, éste es necesario para lograr una mayor penetración de mercado, en donde predomine el lanzamiento de productos y servicios realmente distintos, apoyados en las posibilidades que ofrece la tecnología. El uso de herramientas basadas en las tecnologías de la información debe ser vista como un instrumento de apoyo a las SOFIPO que coadyuve en el cumplimiento de sus objetivos y en la superación de los retos que le establece su modelo de operación actual.

\section{Planteamiento de la hipótesis de trabajo}

Se plantea la hipótesis de que un servicio de valuación de riesgo crediticio soportado con herramientas de credit scoring proporcionado por un tercero; y la formación de capital humano con altos estándares de calidad para operar sistemas para administración de riesgos, son inversiones factibles en términos de costo-eficiencia para las EACP y factibles en términos de costobeneficio para los proveedores del servicio de valuación y consultoría.

Para probar la factibilidad técnica y financiera del servicio de valuación de riesgo crediticio para una EACP se elabora un plan de negocio con los siguientes componentes:

a) un análisis de mercado para determinar la demanda de los servicios propuestos, 
b) se define la política de comercialización del servicio crediticio en un esquema "bajo demanda" evitando costos onerosos a los usuarios,

c) la descripción técnica del proyecto, detallando el algoritmo de credit scoring y la plataforma tecnológica que soporta los servicios de evaluación crediticia,

d) finalmente se realiza la proyección financiera del proyecto y se calculan los indicadores de costo-eficiencia y costo-beneficio para determinar la factibilidad financiera del proyecto.

\section{Descripción del plan de negocio}

Los modelos que se utilizan para la evaluación de los procesos crediticios en el sector financiero bancario son los denominados credit scoring, según (Hand, 1997), son procedimientos estadísticos que se usan para clasificar a aquellos que solicitan crédito, inclusive a los que ya son clientes de la entidad crediticia, en los tipos de riesgo 'bueno' y 'malo'. En sus inicios, durante los años setenta, las aplicaciones del credit scoring se construían con técnicas estadísticas (en particular, con el análisis discriminante). Posteriormente, los métodos empleados evolucionaron a técnicas matemáticas, econométricas y de inteligencia artificial. En cualquier caso, la construcción de toda aplicación del credit scoring se realiza tomando la información del cliente contenida en las solicitudes del crédito, de fuentes internas e, incluso, de fuentes externas de información. El credit scoring estima en el momento en el que se está llevando a cabo la solicitud, cuál será el comportamiento del crédito hasta su vencimiento, atendiendo al riesgo del cliente. Se evalúa a través de un modelo predictivo de comportamiento de pago o reembolso mediante una puntuación que mide el riesgo de un prestatario y/o de laoperación.

Por lo anteriormente descrito, un servicio de valuación de riesgo crediticio tiene como base un conjunto de metodologías y herramientas estadísticas, que deben ser analizadas e implementadas por un equipo de trabajo multidisciplinario con especialistas en finanzas, estadística y desarrolladores de software avanzados.

Los servicios de valuación crediticia son los siguientes:

a) Con base en la información contenida en las solicitudes de crédito y el conocimiento del analista de riesgo del entorno socioeconómico del solicitante, se procesarán automáticamente las solicitudes a 
través de modelos de credit scoring asignándoles probabilidades de incumplimiento y con base en algún umbral se clasificarán en "buenas" o "malas". Lo anterior se define en un esquema de "servicios por demanda", es decir, los clientes de las EACP, no requieren tener instalado los sistemas informáticos y bases de datos en su infraestructura, sino a través de un ambiente WEB realizarán la petición del servicio y como resultado tendrán la clasificación de la solicitud como "buena" o "mala" con su probabilidad de incumplimiento correspondiente. Con lo cual sólo pagarán por los servicios recibidos y no deberán preocuparse por las nuevas versiones del sistema informático ya sea por actualización tecnológica o por mejoras en los métodos de cálculo.

b) Para lograr la administración integral de riesgos eficiente, la cultura financiera es fundamental, por lo que se requieren servicios de acompañamiento y asesoría a las EACP para modelar sus procesos de negocio alineados a las mejores prácticas en la administración de riesgos y a la normatividad en la materia. El entorno incierto y dinámico de los mercados financieros de crédito obliga a las instituciones a mantener capacitados a sus analistas y tomadores de decisiones, en técnicas y herramientas estadísticas y matemáticas que permitan modelar entornos con estas características, por lo que se proporcionarán sistemáticamente programas de actualización y capacitación congruentes con las mejores prácticas de administración de riesgos y factibles con las necesidades propias de las instituciones de crédito.

Hoy en día el sector financiero en México tiene disponible aplicaciones para operar sus procesos crediticios las que cuentan con sistemas con altos estándares de desempeño y seguridad, se puede afirmar que es el sector más aventajado en el uso y aprovechamiento de las tecnologías de la información; no obstante, un alto porcentaje de la tecnología utilizada son adaptaciones de modelos diseñados para instituciones financieras globales y no siempre se adaptan a la realidad del sector financiero mexicano, sobre todo en las entidades que no son bancos ni casas de bolsa, como por ejemplo: sociedades financieras populares, sociedades financieras comunitarias; así como, los organismos de integración financiera rural y PYMES, entre otros; las cuales no cuentan con los recursos presupuestales ni con los recursos humanos especializados para operar sistemas complejos de administración de riesgos, de aquí la justificación para desarrollar un sistema de información, como el que se propone, que apoye a las EACP en la toma de decisiones durante la 
evaluación de créditos y que no les implique erogaciones suntuosas, si no que paguen por servicios requeridos y "a la medida".

Hasta donde se tiene información, no existe en el mercado un producto con estas características, en cambio se prevé una demanda extensa de este tipo de servicios, la demanda se analizará con mayor detalle en la sección siguiente.

\section{Estudio de mercado}

De acuerdo con la información proporcionada por la CNBV a través de su portal, actualmente existen 44 SOFIPO autorizadas por la misma CNBV con activos por 20 mil 318 millones de pesos y con una cartera de 2.7 millones de clientes, de los cuales el $60 \%$ son clientes de la empresa "Libertad", 11\% de "Microemprendedor" y 6\% de "Fincomun". Estos datos muestran una alta concentración del mercado en estas tres instituciones autorizadas, sin embargo, existen instituciones dentro del sector ahorro y crédito popular que se encuentran en proceso de autorización o que operan independientemente de la vigilancia y supervisión de la CNBV.

La Asociación Mexicana de SOFIPO, AMSOFIPO argumenta que, en promedio, el sector integrado a la fecha por 44 entidades de ese tipo debidamente autorizadas, ha crecido en número de clientes (crédito y ahorro) $21 \%$ anual. Resalta que también ha crecido el número de entidades en operación, con un promedio de $13 \%$ anual en el último trienio, y el de sucursales en $26 \%$ anual, con lo que se espera superar las 1200 sucursales al cierre de 2013, distribuidas en todo el país.

A partir de 2010 se observó un crecimiento aproximado del 18\% anual en la cartera de crédito en el sector, que se espera llegue a 20000 millones de pesos al cierre de 2013, mientras la banca obtuvo un aumento de $13 \%$ en este indicador en el último año. Si consideramos préstamos promedio de $4000^{1}$ pesos en este tipo de instituciones, hasta 2013 se estarían evaluando al menos cinco millones de solicitudes de crédito, sin considerar las solicitudes rechazadas, estimando un $40 \%$ de solicitudes rechazadas respecto a las autorizadas, tendríamos un total estimado de siete millones de solicitudes

\footnotetext{
${ }^{1}$ Información obtenida de la publicación del BID “Microfinanzas en América Latina y el Caribe: el sector en cifras 2011", publicado en octubre 2011 (BID (2001).
} 
de crédito que estarían siendo analizadas por los departamentos de crédito para su potencial autorización.

Si se mantiene el crecimiento del $18 \%$ en la cartera de crédito del sector, para los siguientes dos años se tendrían aproximadamente 1.2 millones ( $18 \%$ de siete millones) solicitudes de crédito anuales lo que implica esfuerzos considerables para los departamentos de análisis de crédito, por lo que el contar con un proceso automatizado de evaluación del mismo ayudará a los analistas y ejecutivos a tomar decisiones de manera más eficiente.

En principio, los clientes para el servicio de evaluación que se propone son las $44 \mathrm{SOFIPO}$ autorizadas por la $\mathrm{CNBV}$, aunque dicho servicio también lo requieren aquéllas que están en proceso de autorización o que operan independientemente de la vigilancia de ésta. Hasta julio de 2011 la Secretaría de Economía a través de Fondo Nacional para las Empresas en Solidaridad (FONAES) tenía un padrón de 907 cajas solidarias en todo el país, de las cuales 453 están autorizadas por la CNBV y 454 en proceso de registro, este universo de instituciones también son potenciales clientes de los servicios de evaluación de solicitudes de crédito, en virtud de que se fondearían con recursos fiscales para otorgar microcréditos, bajo las reglas de la Reforma Financiera aprobada en noviembre de 2013. Por tanto, se estima que la cartera de créditos de las instituciones financieras de microcréditos (SOFIPO, SOFOMES, SOCAPS, cajas solidarias, etc.) estaría creciendo a razón del 20 ó $25 \%$ anual a partir de 2015.

Por lo que respecta a productos de análisis de credit scoring en el sector de las microfinanzas es prácticamente nulo, en algunos casos (instituciones nivel IV) ${ }^{2}$ utilizan sistemas diseñados para el sector bancario y para otros (instituciones nivel I, II y III) la evaluación de las solicitudes de crédito se realizan de forma subjetiva y discrecional por los analistas y gerentes de las áreas de crédito.

La conformación del sector de las microfinanzas en México tiene como génesis cubrir un mercado excluido por el sector bancario, por lo que su

\footnotetext{
${ }^{2}$ Nivel de operaciones I: sociedades financieras populares con un monto de activos totales iguales o inferiores a 15 millones de UDIS.

Nivel de operaciones II: sociedades financieras populares con un monto de activos totales superiores a 15 millones e iguales o inferiores a 50 millones de UDIS.

Nivel de operaciones III: sociedades financieras populares con un monto de activos totales superiores a 50 millones e iguales o inferiores a 280 millones deUDIS.

Nivel de operaciones IV: sociedades financieras populares con un monto de activos totales superiores a 280 millones de UDIS.
} 
desarrollo se posiciona por regiones geográficas y por sectores productivos, generando un crecimiento desvinculado de la supervisión y autorización de la autoridad competente y con elevados riesgos tanto para las entidades de crédito como para los ahorradores e inversionistas. En consecuencia, se identifican las siguientes barreras iniciales para un proyecto con estas características:

a) las instituciones no cuentan con procesos documentados

b) cultura financiera limitada

c) silos de información sin estructura

d) no existe incentivos para compartir información, es decir, no existe una central de información sobre el comportamiento crediticio de los acreditados ni tampoco de microfinancieras.

e) instituciones enfocadas a clientes de un solo sector

f) no se cuenta con controles internos

g) gobierno corporativo inexistente

h) sin esquemas de garantías

i) sin voluntad de cambio

j) procesos no automatizados

k) negocios familiares

l) impedimento por parte de la autoridad reguladora

En noviembre de 2013 el poder legislativo aprobó la reforma financiera, la cual considera acciones que motivarán un incremento en el otorgamiento de créditos para el sector de microcréditos, en particular en el sector financiero popular, la reforma propone incrementar los servicios que presta en un esquema de corresponsal bancario, con lo cual estarían en condiciones de obtener mayores beneficios económicos a través de comisiones, pero también una mayor exposición a los distintos riesgos. Lo anterior, fortalece el proyecto de un sistema de riesgos crediticios como el que se propone en este documento.

\section{Descripción del proyecto}

\subsection{Descripción comercial}

El factor crítico de éxito para el proyecto es la cultura financiera, por tanto, resulta necesario llevar a cabo una estrategia de difusión y acompañamiento 
para las instituciones de ahorro y crédito popular, a través de organizar seminarios, pláticas y reuniones con los interesados, para destacar y convencer sobre las ventajas de contar con modelos de administración de riesgos como el que se propone en este trabajo.

\subsection{Política de ventas}

a) para el caso del servicio de evaluación de solicitudes de crédito con herramientas de credit scoring, a través de aplicaciones en ambiente WEB, son servicios por demanda y los costos estarán en función del volumen mensual y el horario de servicio.

b) por lo que respecta al acompañamiento y asesoría se cotizarán por hora y el costo estará en función del nivel del(os) experto(s) requerido(s).

c) finalmente los servicios de capacitación y actualización se organizarán en cursos cortos de una a dos semanas y diplomados que se impartirán con el soporte de alguna institución académica de nivel superior reconocida, los costos estarán en función de la duración y temática a desarrollar.

\subsection{Fórmulas de promoción y publicidad}

Además de la difusión del proyecto en medios impresos y electrónicos, se instrumentarán las siguientes acciones:

a) participación en ferias del sector microcrédito y ahorro popular organizadas por dependencias gubernamentales y asociaciones financieras, nacionales e internacionales

b) creación de una página WEB para clientes con información relevante y servicios de "chats" para atención de preguntas y comentarios

c) creación de aplicaciones para dispositivos móviles con una versión beta del producto

d) creación de una versión académica para el uso en instituciones de enseñanza superior

e) creación de un laboratorio virtual en línea para realizar simulaciones de análisis de riesgo en el sector financiero.

El sector financiero es dinámico, las herramientas y modelos de administración de riesgos también se desarrollan en esta lógica, por tanto, otra ventaja del modelo propuesto de servicios "por demanda" es que las actualizaciones ya sea por cambio tecnológico o por mejoras en las herramientas de cálculo 
del credit scoring es totalmente transparente para el cliente, es decir, cada vez que exista algún cambio, se le notificará al cliente y si está de acuerdo, automáticamente tendrá a su disposición sin costo adicional las mejoras o actualizaciones sin necesidad de hacer modificaciones en sus sistemas o infraestructura tecnológica.

\section{Descripción técnica}

\subsection{Cálculo de credit scoring}

Para plantear un modelo cuya variable respuesta o dependiente sea binaria -ya que las dos situaciones posibles es que el cliente paga (0) o el cliente no paga (1)-, se aplicará un modelo de regresión logística binaria con el objeto de evitar los inconvenientes que presentan los modelos de regresión lineal o de análisis

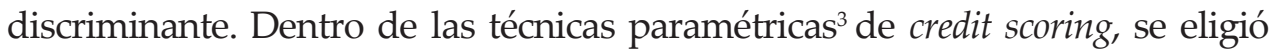
la regresión logística binaria como técnica estadística empleada debido a sus mayores ventajas, entre las cuáles están las siguientes (Salvador y Juan, 2010):

a) las propiedades estadísticas son más adecuadas que las de los modelos lineales en los que, en ocasiones, se obtienen estimadoresineficientes

b) dadas las características del historial crediticio de las SOFIPO, donde la información cualitativa complementa la escasez de variables cuantitativas, la regresión logística binaria admite las variables categóricas con mayor flexibilidad que los modelos lineales

c) permite estimar la probabilidad de impago del crédito según los valores de las variables independientes

d) determina la influencia de cada variable independiente sobre la variable dependiente (pago o impago) según el OR (Odd Ratio o Ventaja). Éste se define como exp $(\beta)$, donde exp es la base de los logaritmos neperianos (una constante cuyo valor es 2.718), y $\beta$ es el valor del parámetro de regresión de la variable independiente en el modelo. Una OR mayor que 1 indica un aumento en la probabilidad del evento de incumplimiento sobre el hecho de pagar cuando la variable explicativa aumenta en una unidad; inversamente, una OR menor que 1 indica lo contrario.

\footnotetext{
${ }^{3}$ Las técnicas paramétricas de credit scoring utilizan una función de distribución o clasificación conocida, al igual que estiman unos parámetros para explicar un determinado suceso, de tal modo que éstos se ajusten a las observaciones de una muestra. Estas técnicas son muy útiles cuando el conjunto de variables siguen una distribución propuesta.
} 


\section{El modelo de regresión logística binaria}

La primera diferencia entre regresión normal y regresión logística es el uso de una variable aleatoria dependiente dicotómica. Este tipo de variable se denomina de Bernoulli, el uso de la regresión normal para este tipo de variables puede violar varios de los supuestos.

En los modelos de regresión lineal se asume que la media condicional de la variable dependiente $\mathrm{y}$, dado la variable independiente $x$, puede ser expresado como una ecuación lineal en $x$, como se observa en la ecuacion 1 .

$$
E(y \mid x)=\beta_{0}+\beta_{1} x
$$

Esta expresión implica que cuando $x$ toma valores entre $(-\infty, \infty)$, ecuación 2 .

$$
0 \leq E(y \mid x) \leq 1
$$

Para establecer una cota de la media condicional, supongamos que se analiza el incumplimiento de los clientes en tarjetas de crédito, para lo cual se incluye como variable independiente el tamaño de las familias y se toma la proporción de incumplimiento para cada tamaño de familia según se muestra en la tabla 1:

\section{Tabla 1}

Análisis de incumplimiento de los clientes de tarjetas de crédito

\begin{tabular}{|cccc|}
\hline $\begin{array}{c}\text { Tamaño } \\
\text { familia }\end{array}$ & \multicolumn{2}{c}{$\begin{array}{c}\text { indicador } \\
\text { incumplimiento }\end{array}$} & \\
\cline { 2 - 3 } & $\mathbf{0}$ & $\mathbf{1}$ & \\
\hline 1 & 9 & 1 & 0.100 \\
2 & 13 & 2 & 0.133 \\
3 & 9 & 3 & 0.250 \\
4 & 10 & 5 & 0.333 \\
5 & 7 & 6 & 0.461 \\
6 & 3 & 5 & 0.625 \\
7 & 4 & 13 & 0.764 \\
8 & 2 & 8 & 0.800 \\
\hline
\end{tabular}

Fuente: elaboración propia. 
En la figura 1 se aprecia que la probabilidad de incumplimiento se aproxima gradualmente a uno cuando se incrementa el tamaño de las familias. Este tipo de curva tiene la forma de una " $S$ " y se le denomina curva logística la cual está determinada por la ecuación 3:

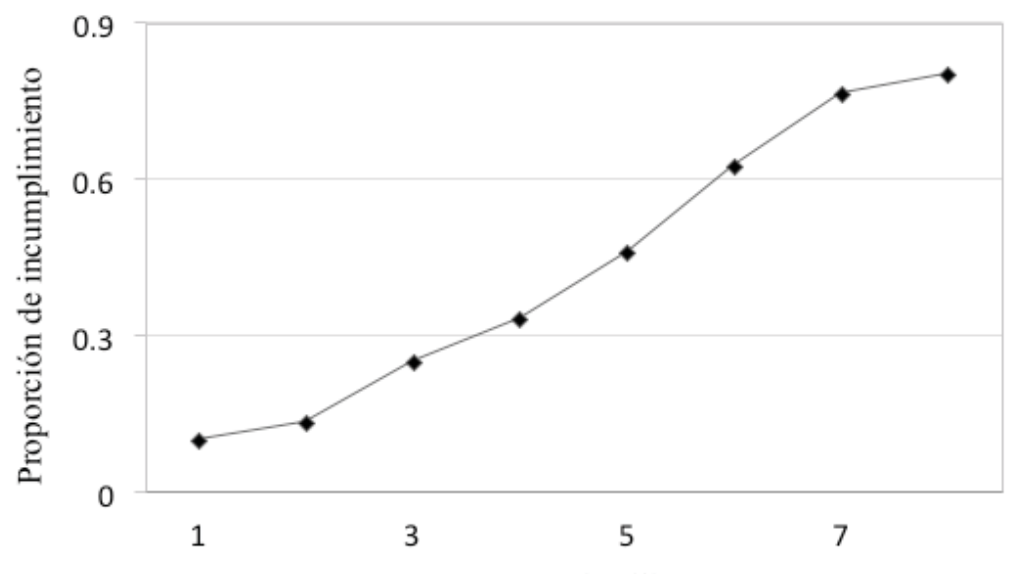

Tamaño familias

Figura 1. Probabilidad de incumplimiento de los clientes de tarjetas de crédito

Fuente: elaboración propia.

$$
p=\pi(x)=\frac{e^{\beta_{0}+\beta_{1} x}}{1+e^{\beta_{0}+\beta_{1} x}}
$$

donde, $\pi(x)$ representa $E(y \mid x)$ es decir, la probabilidad de incumplimiento. Reasignando términos, tenemos.

$$
\frac{\pi(x)}{1-\pi(x)}=e^{\beta_{0}+\beta_{1} x}
$$

Además

$$
\begin{gathered}
\operatorname{In}\left(\frac{\pi(x)}{1-\pi(x)}\right)=\beta_{0}+\beta_{1} x \\
G(x)=\operatorname{In}\left(\frac{\pi(x)}{1-\pi(x)}\right)=\beta_{0}+\beta_{1} x
\end{gathered}
$$


$G(x)$ es denominada la transformación logística la cual posee muchas de las propiedades deseables de un modelo de regresión lineal; es lineal en sus parámetros, puede ser continua y tomar valores entre $(-\infty, \infty)$ dependiendo del dominio de $x$.

En un modelo de regresión lineal se asume que la variable dependiente puede ser expresada como $y=\beta_{0}+\beta_{1} x+\varepsilon$. Donde $\varepsilon$ se distribuye con media cero y varianza constante. Considerando a la variable dependiente como binaria dado el valor de $x$, ahora la expresamos como $y=\pi(x)+\varepsilon$. El error $\varepsilon$ tiene dos posibles valores, si $y=1$, entonces $\varepsilon=1-\pi(x)$ con probabilidad $\pi(x)$, si $y=0$, entonces $\varepsilon=-\pi(x)$ con probabilidad 1- $\pi(x)$. Así, $\varepsilon$ se distribuye con media cero y varianza $\frac{\pi(x)}{1-\pi(x)}$. Por tanto, como el término de error se distribuye de esta manera, se tiene que la distribución condicional de la variable dependiente es una distribución binomial con una probabilidad dada por la media condicional $\pi(x)$.

Para ajustar un modelo de regresión logística $p=\pi(x)=\frac{e^{\beta_{0}+\beta_{1} x}}{1+e^{\beta_{0}+\beta_{1} x}}, \quad$ a un conjunto de datos se requiere estimar los valores para los parámetros desconocidos $\beta_{0} \mathrm{y} \beta_{1}$, de forma que minimicen el cuadrado de la suma de los errores (SSE). Con algunos modelos como el de regresión logística no existe una solución matemática que produzca una expresión explícita para la estimación de los parámetros por mínimos cuadrados ordinarios. El método que utilizaremos es el de máxima verosimilitud, el cual produce valores para los parámetros desconocidos que maximizan la probabilidad de que sean obtenidos de los datos observados.

Si $y$ toma valores de 0 ó 1 , la expresión para $p=\pi(x)=\frac{e^{\beta_{0}+\beta_{1} x}}{1+e^{\beta_{0}+\beta_{1} x}}$, proporciona la probabilidad condicional de que $y=1$ dado $x, P(y=1 \mid x)$. Se sigue que $1-\pi\left(x_{i}\right)$ es la $P(y=0 \mid x)$, para alguna observación $\left(x_{i}, y_{i}\right)$ donde $y_{i}=1$, la contribución a la función de verosimilitud es $\pi\left(x_{i}\right)$ y donde $y_{i}=0$, la contribución a la función de verosimilitud es $1-\pi\left(x_{i}\right)$ lo cual puede expresarsecomo:

$$
\pi\left(x_{i}\right)^{y i}\left[1-\pi\left(x_{i}\right)\right]^{1-\mathrm{yi}}
$$

Donde las observaciones son independientes, así la función de verosimilitud se obtiene con el producto de los términos dados en la expresión anterior. 


$$
L(\beta)=\operatorname{In}\left(L(\beta)=\sum_{i=1}^{\mathrm{n}}\left\{y_{i} \operatorname{In}\left[\pi\left(x_{i}\right)\right]+\left(1-y_{i}\right) \operatorname{In}\left[1-\pi\left(x_{i}\right)\right]\right\}\right.
$$

En regresión logística, minimizar el logaritmo de la función de verosimilitud genera ecuaciones que no son lineales en los parámetros, entonces se utilizan métodos numéricos para aproximar la solución. En el ejemplo de incumplimiento de tarjetas de crédito, las posibilidades de incumplimiento para una familia de tamaño ocho se obtienen de la siguiente manera:

$$
\begin{aligned}
\text { odds }= & \frac{p}{1-p} \\
& =\frac{0.8}{0.2}=4
\end{aligned}
$$

Es decir que la relación es de 4 a 1. Ahora, la transformación logística se utiliza para la regresión logística. Esto es precisamente el logaritmo natural de las posibilidades de incumplimiento:

$$
\begin{gathered}
G(x)=\operatorname{In}\left(\frac{\pi(x)}{1-\pi(x)}\right)=\beta_{0}+\beta_{1} x \\
G(x)=\operatorname{In}(\text { Odds }) \\
\operatorname{In}\left(\frac{\pi(x)}{1-\pi(x)}\right)=\beta_{0}+\beta_{1} x, \\
\operatorname{In}\left(\frac{p}{1-p}\right)=\beta_{0}+\beta_{1} x, \\
\frac{p}{1-p}=e^{\beta_{0}+\beta_{1} x,} \\
p=\pi(x)=\frac{e^{\beta_{0}+\beta_{1} x}}{1+e^{\beta_{0}+\beta_{1} x}} .
\end{gathered}
$$

El proceso central de los modelos de credit scoring estará alojado en un servidor de alta disponibilidad y canales seguros de comunicación. Además, si el cliente lo requiere se tendrán bases de datos centralizadas para resguardar la información de sus solicitudes bajo los estándares de privacidad que establezca la autoridad competente, garantizando la disponibilidad, confidencialidad e integridad de su información. El tener información en una sola base de datos permitirá a las entidades aprovechar la información del sector la cual se conformará con la información que provean todas las EACP y que suscriban un convenio de colaboración interinstitucional, lo anterior 
puede ayudar a corregir las fallas del mercado de crédito en el sector popular asociadas con información asimétrica.

$\mathrm{Al}$ inicio, la demanda de servicios no requiere inversiones fuertes para el alojamiento del proceso de credit scoring, sin embargo, de acuerdo con la demanda esperada del proyecto para el 2015, que se estima en un 10 a $20 \%$ de la oferta del mercado, se debe considerar una inversión para la adquisición o arrendamiento de infraestructura tecnológica de punta que garantice la atención del volumen de solicitudes de evaluación de crédito de diversas EACP.

\subsection{Arquitectura tecnológicapropuesta}

El propósito de esta sección es proporcionar una visión comprensible de la arquitectura general del sistema de información propuesto, utilizando diversas vistas de la arquitectura para ilustrar diferentes aspectos del sistema.

\section{Representación general de la arquitectura propuesta}

Dentro del sistema de información propuesto se contempla un modelo de aplicación distribuida de tipo cliente servidor, en el cual distintos equipos cliente podrán acceder a la aplicación a través de internet. La aplicación instalada en un servidor WEB centralizado será capaz de dar servicio a los equipos cliente a través del sistema propuesto mediante una interfaz gráfica de usuario con la cual se interactuará en todo momento.

Los datos que se generan y se consumen por el sistema propuesto son almacenados en otro equipo de cómputo denominado servidor de base de datos al cual tendrá acceso únicamente el servidor WEB y el servidor de servicios (el cual se menciona más adelante), imposibilitando la interacción directa del usuario con los datos para procurar su confidencialidad e integridad.

Tal y como se mencionó anteriormente hasta el momento se prevé la aplicación de la técnica de regresión logística, entendido como un servicio que clasifica una solicitud de crédito ingresada como "buena" o "mala" con su probabilidad de incumplimiento correspondiente. Posteriormente se pretende incorporar en una segunda versión del sistema informático, los servicios de clasificación basados en técnicas más eficientes como las redes bayesianas o algoritmos genéticos. Debido a lo anterior y con el objetivo de dotar de escalabilidad al sistema propuesto, cada uno de los servicios 
de credit scoring proporcionados por el sistema residirán en otro servidor que tendrá un acceso restringido y cuyo funcionamiento se basará en el uso de servicios Web a través del estándar XML/SOAP.

Las principales ventajas del uso de este tipo de arquitectura son las siguientes:

a) centralización del control de los recursos, datos y accesos

b) facilidad de mantenimiento y actualización del lado del servidor:esto es porque el lado del servidor se puede mantener o actualizar fácilmente. Por ejemplo, una actualización se aplica a un único servidor, pero los beneficios los obtienen múltiples clientes generalmente sin necesidad de que éstos actualicen nada

c) toda la información es almacenada en el lado del servidor, que suele tener mayor seguridad que los clientes

d) interoperabilidad entre aplicaciones de software independientemente de sus propiedades o de las plataformas sobre las que seinstalen

\subsection{Seguridad de la arquitectura propuesta}

Se pretende dotar de distintos niveles de seguridad al sistema propuesto a través de los siguientes elementos:

a) proveer un ingreso al sistema mediante un modelo seguro de usuarios y contraseñas

b) instalación de una rutina de programación que haga las funciones de filtro de entradas de comunicación al servidor WEB para evitar ataques de tipo semántico, de rutinas cruzadas y de peticiones HTTP falsificadas

c) configuración de un Firewall físico y lógico que permita la comunicación únicamente a los equipos conectados en redes autorizadas

d) uso de certificados de seguridad para el consumo de servicios de credit scoring

\subsection{Vista de implementación}

La vista de implementación seguirá el patrón de diseño MVC (modelovista-controlador). Este patrón plantea la separación del problema en tres capas: la capa modelo, la capa controlador y la capa vista. 
a) El modelo es la representación de la información con la cual el sistema opera, por lo tanto gestiona todos los accesos a dicha información, tanto consultas como actualizaciones. Envía a la 'vista' aquella parte de la información que en cada momento se le solicita para que sea mostrada. Las peticiones de acceso o manipulación de información llegan al 'modelo' a través del 'controlador'.

b) El controlador responde a acciones del usuario e invoca peticiones al 'modelo' cuando se hace alguna solicitud sobre la información. Se puede decir que el 'controlador' hace de intermediario entre la 'vista' y el 'modelo'.

c) La vista presenta el 'modelo' en un formato adecuado para interactuar por tanto requiere de dicho 'modelo' la información que debe representar como salida.

\subsection{Vista de proceso}

En esta vista se presenta el proceso en el cual se basará el sistema propuesto por medio del conjunto de tareas relacionadas lógicamente con el objetivo de evaluar la solicitud de microcrédito. El proceso propuesto es mostrado tomando en cuenta tres niveles de granularidad como se muestra en la figura 2 :

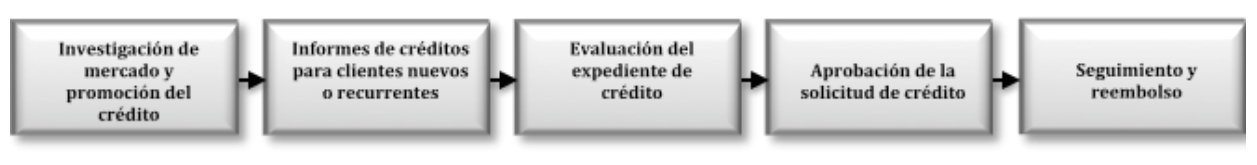

Figura 2. Modelo de procesos de otorgamiento de crédito.

Primer nivel 


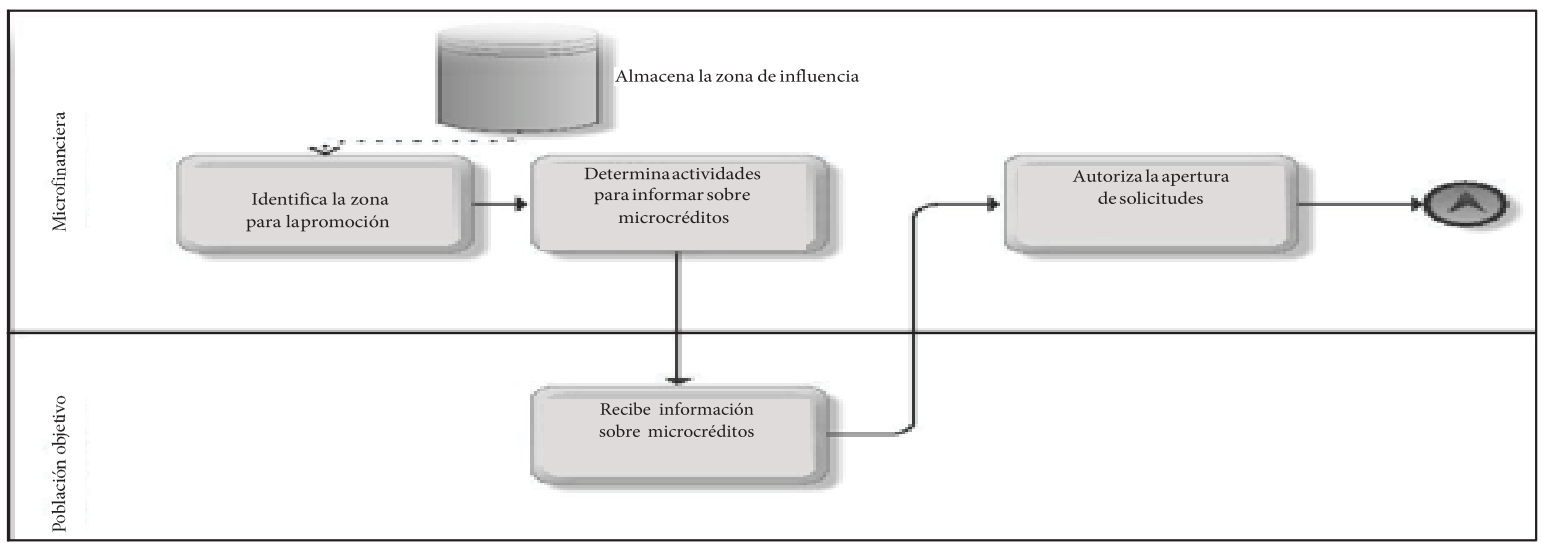

Segundo nivel. Investigación de mercado y promoción del crédito.

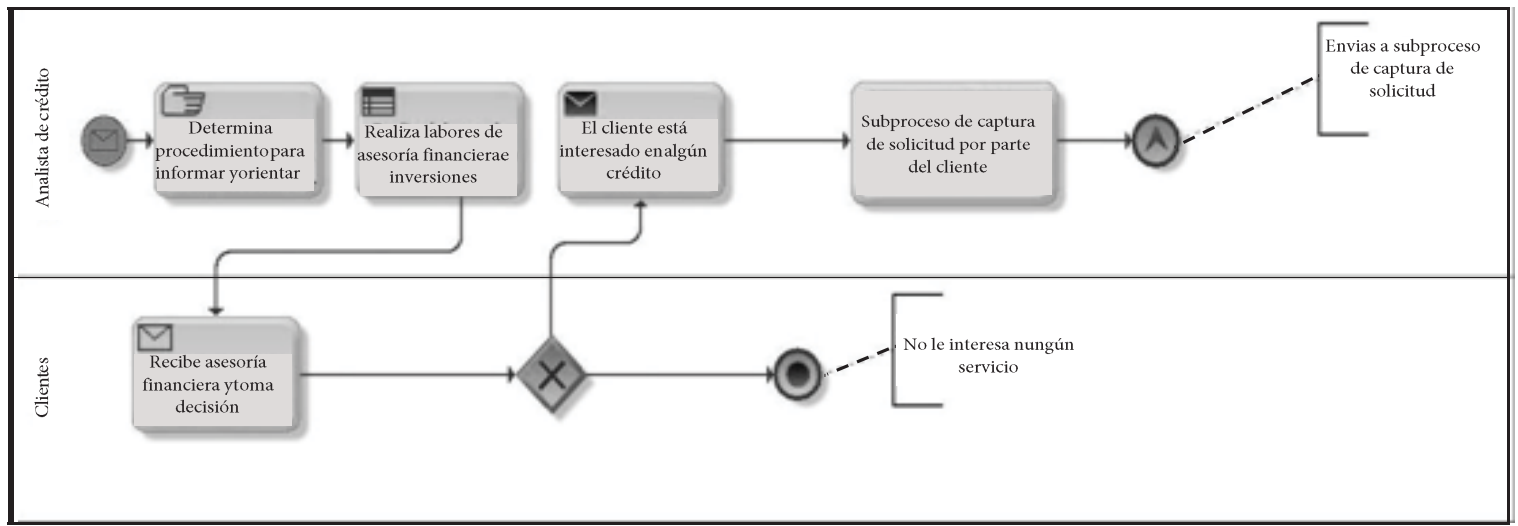




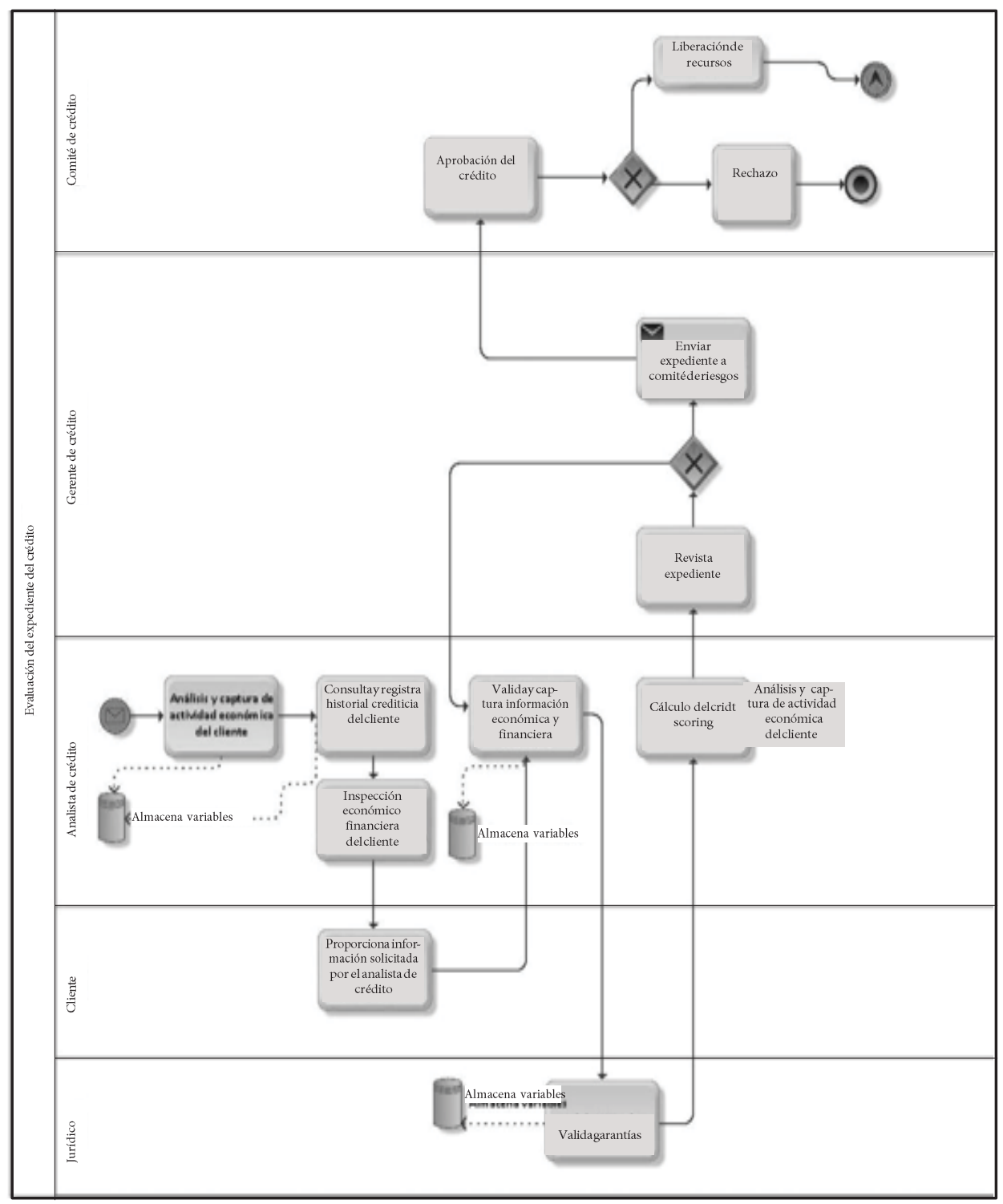

Conclusión: segundo nivel. 

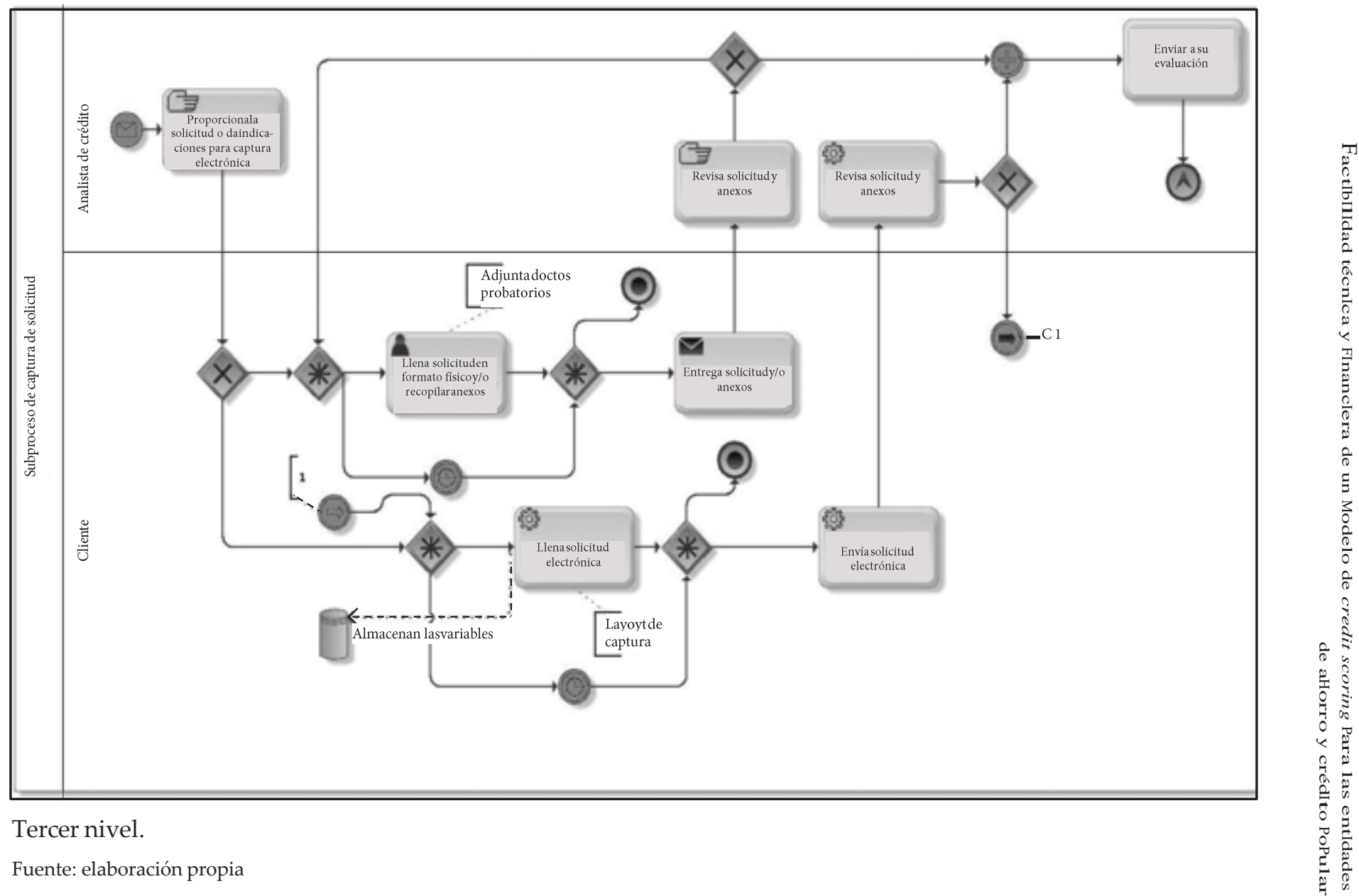

Tercer nivel.

Fuente: elaboración propia 


\section{Administración del proyecto}

\subsection{Alcance del proyecto}

En este apartado se proporcionan algunas prácticas internacionales para la administración de proyectos, las cuales garantizan el cumplimiento de los objetivos y metas de un proyecto.

El proyecto incluye:

1. Diseñar y desarrollar un software informático para identificar, medir y cuantificar los riesgos crediticios que enfrentan las entidades de ahorro y crédito popular.

2. Formar capital humano con altos estándares de calidad para operar sistemas para administración de riesgos.

El proyecto NOincluye:

1. Se supone que los clientes de las SOFIPO tienen la infraestructura tecnológica para acceder a internet y consultar el sistema. El proyecto no contempla ningún tipo de equipamiento, dotación, capacitación, etc. en cuanto a infraestructura.

2. El proyecto no incluye la captura, formateo, generación ni ningún tipo de manipulación de la información histórica correspondiente a las operaciones y transacciones aprobadas yrechazadas por cada cliente.

\subsection{Planeación del proyecto}

Con base en la situación actual; se propone conformar un equipo interdisciplinario con experiencia en proyectos similares que agreguen valor por su conocimiento de los procesos y tecnología. Ellos, en conjunto con los actores clave de las EACP deberán identificar, analizar y elegir la mejor solución que otorgue valor a la operación de las EACP.

Gran parte del éxito de esta implantación depende de las definiciones y análisis de proceso obtenido como parte del diagnóstico inicial. Es por esto que se requiere de los actores clave que tengan pleno conocimiento de la gestión del control y operación del área.

Considerando lo anterior, la planeación del proyecto que incluirá la metodología a utilizar, la definición de las etapas de desarrollo, la asignación detallada de actividades y responsabilidades, la definición de 
roles, los mecanismos de control, entre otros; se detallará al momento de iniciar el proyecto (tablas 2 y 3 ).

\subsection{Recursos iniciales del proyecto}

\section{Tabla 2}

Resumen de recursos iniciales requeridos

\begin{tabular}{|lcc|}
\hline \multicolumn{1}{|c}{ Tipo de recursos } & cantidad & $\begin{array}{c}\text { total } \\
\text { pesos mexicanos }\end{array}$ \\
\hline recursos humanos & 6 & 515000.00 \\
gastos de inversión & 21 & 79000.00 \\
soporte & 4 & 140000.00 \\
recursos humanos para & 3 & 114000.00 \\
la operación & & 848000.00 \\
\hline
\end{tabular}

Fuente: elaboración propia. 


\section{Tabla 3}

Detalle de recursos iniciales requeridos

\begin{tabular}{|c|c|c|c|c|}
\hline Concepto & Descripción & $\begin{array}{c}\text { costo } \\
\text { unitario }\end{array}$ & cantidad & $\begin{array}{c}\text { Total } \\
\text { pesos } \\
\text { mexicanos }\end{array}$ \\
\hline analista & $\begin{array}{l}\text { maestría en finanzas } \\
\text { o ciencias }\end{array}$ & 50000.00 & 1 & 50000.00 \\
\hline programador & $\begin{array}{l}\text { licenciatura o maestría en } \\
\text { ciencias computacionales }\end{array}$ & 100000.00 & 2 & 200000.00 \\
\hline líder técnico & $\begin{array}{l}\text { maestría o doctorado } \\
\text { en ciencias }\end{array}$ & 120000.00 & 1 & 120000.00 \\
\hline líder de proyecto & doctor en ciencias & 120000.00 & 1 & 120000.00 \\
\hline \multirow[t]{2}{*}{ diseño } & diseñador WEB & 25000.00 & 1 & 25000.00 \\
\hline & & \multicolumn{3}{|c|}{ Total recursos humanos 515000.00} \\
\hline seminarios & promoción del proyecto & 25000.00 & 1 & 25000.00 \\
\hline \multirow[t]{2}{*}{ viáticos } & $\begin{array}{l}\text { levantamiento de } \\
\text { información }\end{array}$ & 3000.00 & 18 & 54000.00 \\
\hline & & \multicolumn{3}{|c|}{ Total gastos de inversión 79000.00} \\
\hline equipo de cómputo & equipo portátil & 30000.00 & 3 & 90000.00 \\
\hline \multirow[t]{2}{*}{ software estadístico } & $\begin{array}{l}\text { licenciamiento de software } \\
\text { especializado }\end{array}$ & 50000.00 & 1 & 50000.00 \\
\hline & & \multicolumn{3}{|c|}{ Total soporte 140000.00} \\
\hline calificada (cursos) & instructores & 20000.00 & 1 & 20000.00 \\
\hline calificada (diplomados) & $\begin{array}{l}\text { profesionales con } \\
\text { posgrado }\end{array}$ & 40000.00 & 1 & 40000.00 \\
\hline \multirow[t]{2}{*}{ no calificada } & apoyo administrativo & 54000.00 & 1 & 54000.00 \\
\hline & \multicolumn{4}{|c|}{ Total recursos humanos (operación) 114000.00} \\
\hline
\end{tabular}

Fuente: elaboración propia.

\section{Análisis costo-eficiencia}

El costo por servicio de la propuesta implica para las EACP erogaciones sólo por los servicios solicitados, la evaluación crediticia disminuye la probabilidad de otorgar un crédito a clientes "malos", lo cual tiene efectos más severos que si no se otorga el crédito a personas "cumplidas". Por tanto, se 
producirán beneficios (disminución de probabilidad de otorgar créditos en default, disminución de tiempos de respuesta y disminución de costos de operación) en las siguientes actividades del proceso de otorgamiento del crédito en las EACP:

1. En el proceso actual los solicitantes de crédito tienen que esperar días o incluso semanas, para la aprobación de préstamos. Los analistas de crédito utilizan su propio juicio para evaluar a los solicitantes, pero la información asimétrica entre prestatarios y prestamistas es tan aguda en el mercado de crédito para la pequeña empresa, que se presentan sesgos en el análisis de crédito y discrecionalidad en lugar de una evaluación sistemática de los riesgos. Las asimetrías de información también inhibe la competencia en el mercado de préstamos a las PyMEs.

2. Instituciones igualmente especializadas en crédito de banca comunal, con promedios de crédito bajos y de un tamaño comparables, registran costos operacionales equivalentes a casi la mitad de los que se presentan en las instituciones de microfinanzas (IMF) del mercado mexicano, una explicación pueden ser las diferencias en la productividad de los analistas. La falta de capacitación y la alta rotación de los analistas, al punto de que incluso las entidades nuevas, en donde la rotación es superior al $100 \%$ en un año. Obviamente, la rotación y la "piratería de los analistas" entre competidores se facilita en mercados con fuerte competencia, y en donde la metodología de evaluación de riesgo no requiere una evaluación de la capacidad de pago de los deudores, sino la habilidad para conformar grupos de deudores.

3. La falta de escala y de eficiencia está en el centro de la explicación de por qué persisten las elevadas tasas de interés del mercado mexicano aun cuando se precia de ser un mercado muy competitivo. A esto habría que agregar el estímulo hacia el fondeo público orientado a que las instituciones de microfinanzas lleven sus operaciones a las áreas rurales de menos de 50000 habitantes, lo cual podría estar provocando excesivos gastos en instituciones mayoritariamente débiles y poco sostenibles, para atender mercados rurales más riesgosos, complejos y costosos.

Los anteriores problemas pueden ocurrir en una entidad, pero es aún más grave cuando se dan en un mercado entero que se especializa en 
ofrecer un sólo producto de crédito. Esta situación entraña riesgos significativos, lo que está pasando en el mercado mexicano, ante la limitada oferta de productos adecuados a su capacidad de pago, es que muchos clientes llegan a ser parte de varios bancos comunales para suplir sus necesidades de financiación. Esto no sólo resquebraja la vinculación del cliente con una institución en particular -que es lo que algunos autores han identificado como el mayor incentivo moral por pagar un crédito- sino que le implica al cliente asumir unos costos elevados, por las altas tasas deinterés típicas del mercado mexicano y también porque debe asumir varias veces los elevados costos de transacción que implica estametodología.

La diversificación de productos y de metodologías de evaluación de riesgo es el principal reto que enfrenta la industria de microfinanzas de México.

\section{Análisis costo-beneficio}

A continuación se presentan los resultados de la proyección financiera considerando que se atendería a un $10 \%$ de la demanda de este tipo de servicios, en un horizonte de cinco años, el detalle de costos unitarios y tarifas de servicios se omiten debido a que el proyecto está en proceso de evaluación e implantación y proporcionar estos datos pondrían en riesgo su viabilidad (tabla 4 ). 
Tabla 4

Proyección financiera del proyecto

\begin{tabular}{|c|c|c|c|c|c|c|}
\hline Concepto & 2013 & 2014 & 2015 & 2016 & 2017 & 2018 \\
\hline \multicolumn{7}{|l|}{ ventas } \\
\hline servicios de evaluación & & 720000 & 1440000 & 2700000 & 3600000 & 4500000 \\
\hline asesorías y acompañamiento & & 144000 & 288000 & 1080000 & 1440000 & 1800000 \\
\hline cursos de capacitación & & 72000 & 144000 & 540000 & 720000 & 900000 \\
\hline diplomados & & 40000 & 75000 & 100000 & 150000 & 200000 \\
\hline Porcentaje del mercado & & $2 \%$ & $4 \%$ & $6 \%$ & $8 \%$ & $10 \%$ \\
\hline $\begin{array}{l}\text { Porcentaje de asesorías con } \\
\text { respecto al total de servicios }\end{array}$ & & $20 \%$ & $20 \%$ & $40 \%$ & $40 \%$ & $40 \%$ \\
\hline $\begin{array}{l}\text { Porcentaje de cursos con } \\
\text { respecto las asesorías }\end{array}$ & & $50 \%$ & $50 \%$ & $50 \%$ & $50 \%$ & $50 \%$ \\
\hline Participación en diplomado & & 8 & 15 & 20 & 30 & 40 \\
\hline Entradas de efectivo & 0 & 976000 & 1947000 & 442000 & 5910000 & 7400000 \\
\hline seminarios & 25000 & 25000 & 25000 & & & \\
\hline viáticos & 50000 & 50000 & 50000 & 50000 & 50000 & 50000 \\
\hline apoyo técnico (diseño) & 12250 & & & & & \\
\hline \multicolumn{7}{|l|}{ Equipo de cómputo } \\
\hline \multicolumn{7}{|l|}{ pc } \\
\hline \multicolumn{7}{|l|}{ servidores } \\
\hline laptop & 90000 & & & & & \\
\hline Software & 50000 & & & & & \\
\hline \multicolumn{7}{|l|}{ Mano de obra calificada } \\
\hline programadores & 210000 & 52500 & & 52500 & & 52500 \\
\hline analista & 50000 & 25000 & 25000 & 25000 & 25000 & 25000 \\
\hline líder técnico & 120000 & & & & & \\
\hline líder de proyecto & 120000 & & & & & \\
\hline \multicolumn{7}{|l|}{ mano de obra operación } \\
\hline calificada para cursos & & 20000 & 20000 & 20000 & 20000 & 20000 \\
\hline calificada para diplomados & & 40000 & 40000 & 40000 & 40000 & 40000 \\
\hline no calificada & & 54000 & 54000 & 54000 & 54000 & 54000 \\
\hline \multicolumn{7}{|l|}{ Otros gastos } \\
\hline oficina & & & 1500 & 1500 & 1500 & 1500 \\
\hline \multicolumn{7}{|l|}{ Renta oficina } \\
\hline Salida efectivo & 727250 & 266500 & 215500 & 243000 & 190500 & 243000 \\
\hline Flujo de efectivo neto & -727250 & 709500 & 1731500 & 4177000 & 5719500 & 7157000 \\
\hline Factor de descuento & 1.000 & 0.870 & 0.756 & 0.658 & 0.572 & 0.497 \\
\hline Flujo descontado & -727250 & 616957 & 1309263 & 2746445 & 3270143 & 3558294 \\
\hline
\end{tabular}

Fuente: elaboración propia.

\begin{tabular}{|rc|}
\hline VAN & 10773851 \\
TIR & $192 \%$ \\
Tasa de descuento & $15 \%$ \\
\hline
\end{tabular}


De acuerdo con los resultados de la proyección, el proyecto es financieramente factible ya que tiene un valor presente positivo, una tasa de rendimiento alta y un periodo de recuperación de la inversión dentro de los dos primeros años.

Lo anterior se sustenta en que para su instrumentación y operación los recursos requeridos son mínimos, una vez desarrollada e implantada la plataforma tecnológica y los aplicativos, el servicio de evaluación de las solicitudes de crédito se proporciona en forma automática sin la intervención del personal responsable de la custodia y operación de la plataforma.

No obstante que es un proyecto factible en términos del costo-beneficio, desde el enfoque de las entidades de ahorro y crédito popular y rentable como proyecto de inversión para el proveedor del servicio, como se mostró en ésta y la sección anterior, se debe profundizar el análisis los factores de riesgo y barreras de entrada para este tipo deiniciativas.

Para tener un análisis integral de los riesgos del proyecto se requiere realizar un diagnóstico sobre la operación actual de las EACP a través de un levantamiento de información con una muestra representativa que incluya por lo menos nueve instituciones financieras que otorguen servicios crediticios al sector popular, tres de la región norte del país, tres de la región centro y tres de la región sur. Con esta información se estaría en condiciones para definir un esquema de riesgos en función de las ganancias y rentabilidad esperadas, en este trabajo no está considerado este tipo de análisis, por lo que se propone para siguientes investigaciones un análisis de riesgos para contar con elementos suficientes para una exitosa administración del proyecto.

\section{Conclusiones}

Un modelo de credit scoring con las características propuestas en este trabajo promueve la eficiencia durante las distintas fases y actividades del otorgamiento de créditos, entendiendo a la eficiencia en términos de la disminución de costos de operación, disminución de tiempos de respuesta y sobretodo disminuir la probabilidad de otorgar créditos "malos". Lo anterior genera información disponible en cualquier momento para la toma de decisiones con bases estadísticas sólidas, además de estandarizar los procesos de evaluación lo que eventualmente impactará al mercado de microcrédito en la disminución de las tasas de interés.

Colateralmente, con la información que ingresa al sistema de evaluación y previo consenso entre las instituciones de ahorro y crédito popular, es 
posible sentar las bases para conformar una base de datos inicial para un sistema de información crediticia para este sector, el cual ayudaría enormemente a disminuir la información asimétrica característica del mercado de crédito de las micro-finanzas.

\section{Referencias}

AMSOFIPO (s.f.). http://www.amsofipo.mx/.

BID (2011). Microfinanzas en América Latina y el Caribe: el sector en cifras. DF, Banco Interamericano de Desarrollo, pp. 19.

Consultores, M. (2011). Microfinanzas en México. Corporación Andina de Fomento.

Hand, D. J. (1997). "Statistical Classification Methods in Costumer Credit Scoring: A review". Journal of the Royal Statistical Association, 160, pp. 523-542.

Institute, P. M. (2004). PMBok, PMI.

P., H. A. (2011). "credit scoring, Statistical Techniques and Evaluation Criteria: A review of literature". Intelligent Systems in Accounting, Finance and Management, 18 (2-3), pp. 59-88.

Pagos, B. I. (2004). BASILEA II. BI., pp. 203.

Salvador, R., y Juan, L. (2010). Un modelo de Credit Scoring para instituciones de microfinanzas en el marco de Basilea II. Journal of Economics, Finance and Administrative sciense, 37, pp. 89-124

Valores, C. N. (2001). Ley de Ahorro y Crédito Popular. Méxido DF:CNVB. 